\title{
Ionoluminescence and photoluminescence in swift heavy ion-irradiated $\mathrm{Al}_{2} \mathrm{SiO}_{5}$
}

\author{
H. Nagabhushana ${ }^{\mathrm{a}}$, B.N. Lakshminarasappa ${ }^{\mathrm{a}, *}$, Fouran Singh $^{\mathrm{b}}$, D.K. Avasthi ${ }^{\mathrm{b}}$ \\ ${ }^{a}$ Department of Physics, Bangalore University, Bangalore-560 056, India \\ ${ }^{\mathrm{b}}$ Nuclear Science Centre, Aruna Asaf Ali Marg, New Delhi-110 067, India
}

Received 21 October 2002; received in revised form 14 February 2003; accepted 8 May 2003

\begin{abstract}
Ionoluminescence (IL) and photoluminescence (PL) of aluminum silicates irradiated with $100 \mathrm{MeV}$ swift $\mathrm{Ni}^{+8}$ ions with fluences in the range $7.5 \times 10^{10}-1.0 \times 10^{14}$ ions $/ \mathrm{cm}^{2}$ have been studied. A pair of IL bands with peaks at $\sim 775$ and $875 \mathrm{~nm}$ are recorded in both natural crystalline and pelletized kyanite samples. And, a pair of sharp as well as strong PL emission bands with peaks at $\sim 688.7$ and $705.9 \mathrm{~nm}$ along with a broad emission band in the region 710-900 $\mathrm{nm}$ are recorded in these samples with an excitation of $442 \mathrm{~nm}$ laser beam. It is found that both IL and PL intensities decrease with increase of ion fluence. The decrease of IL is attributed to amorphization of the material.
\end{abstract}

(C) 2003 Published by Elsevier Ltd.

Keywords: Ionoluminescence; Photoluminescence; Kyanite; Ion irradiation

\section{Introduction}

Ionoluminescence, also known as ion beam-induced luminescence (IBIL) is a phenomenon of emission of light when energetic ions interacting with solid and it provides information about the chemical form of elements. Also, it allows the detection of rare earth elements in the host materials with a minimum detection level of a few ppm. In material sciences, the IL methods can be applied to study the intrinsic and extrinsic luminescence phenomena and is capable of micro-characterizing various inorganic materials, including those of geological and synthetic ones. Detailed theory on ionoluminescence (IL) and material modifications due to energetic ion interaction with matter is described by Yang et al. (1993).

Kyanite is an aluminum silicate $\left(\mathrm{Al}_{2} \mathrm{SiO}_{5}\right)$ crystallizes in triclinic and it is usually transparent to translucent, blue in color and generally contains impurities such as iron. They are promising materials for high temperature applications

\footnotetext{
* Corresponding author. Tel.: +91-80-6748-145; fax: +91-80321-9295.

E-mail address: bnlnarasappa@rediffmail.com (B.N. Lakshminarasappa).
}

because of its low thermal conductivity. The system has been of primary interest of petrologists and experimentalists because of its applicability in both geologic thermometers and/or barometers (Dana and Ford, 1962). Literature revealed that IL and photoluminescence (PL) studies on aluminum silicates are very limited. In this paper, ion-induced damage in kyanite have been studied by means of IL, PL and infrared spectroscopy (IR) techniques and the results obtained are presented.

\section{Experimental}

Kyanite single crystals (I) each of $\sim 1 \mathrm{~mm}$ thickness and $45 \mathrm{~mm}^{2}$ area are obtained by cleaving the long thin block of natural kyanite procured from Indian Bureau of Mines, Bangalore. The powder form of kyanite (II) are prepared by crushing big blocks and grinding using pestle and mortar. The grain size of kyanite used in the present work is in the range $75-53 \mu \mathrm{m}$. Pellets (III) each of $6 \mathrm{~mm}$ diameter and $1 \mathrm{~mm}$ thickness are prepared by applying a pressure of 6 tones per ram using a home-made pelletizer at room temperature (RT). The pellets and single crystals are irradiated with $100 \mathrm{MeV} \mathrm{Ni}^{+8}$ swift ions (from 15UD Pelletron) 
at Nuclear Science Center, New Delhi. The detailed experimental setup for online IL and in situ PL measurements using Michelle-900 Spectrograph and CCD are described elsewhere (Singh et al., 2001). The IR absorption are made using Pye Unicam SP3-300 Spectrophotometer.

\section{Results and discussion}

Fig. 1 shows the IL spectra of kyanite single crystals (I) irradiated with $100 \mathrm{MeV} \mathrm{Ni}^{+8}$ swift ions for fluences in the range $0.75-3.00 \times 10^{11}$ ions $/ \mathrm{cm}^{2}$. A pair of IL bands with peaks at about 775 and $875 \mathrm{~nm}$ along with a pair of sharp peaks in the range $680-720 \mathrm{~nm}$ are recorded. Also, similar results are obtained in the case of pelletized kyanite (III), irradiated with the same amount of ion fluence as shown in Fig. 2. The IL intensity in crystalline as well as pelletized kyanite samples are found to be almost same. It is observed that IL intensity is found to be very large at the early stage of ion beam irradiation of a fresh crystal but, at later stages, the intensity starts diminishing with swift heavy ion irradiation. Further, it is found that the IL intensity at the peaks of the IL bands in both crystalline and pelletized kyanite are found to decrease with increase of ion fluence (results for the $775 \mathrm{~nm}$ IL peak are shown in the inset of Fig. 2). The pair of sharp peaks observed in the range $680-720 \mathrm{~nm}$ are attributed to luminescence centers activated by $\mathrm{Fe}^{3+}$ ions. The characteristics of the center giving rise to sharp emission in kyanite might be due to the fact that optically active electron involved in the luminescence emission is within 4f shell which is partially shielded by the outer most electrons $3 \mathrm{~s}^{2}, 3 \mathrm{p}^{6}$ (Homman et al., 1994). The decrease in IL yield might be due to increase in $\mathrm{Fe}^{3+}$ concentration caused by $\mathrm{Ni}^{+8}$ ions irradiation. If the concentration of impurities including rare-earth elements (REEs) are increased in the kyanite, the crystal parameters may change and this play an important role in the extrinsic luminescence processes. Some impurity ions like $\mathrm{Fe}^{3+}$ and $\mathrm{Ni}^{2+}$ usually act as luminescence quenchers (Malmqvist et al., 1996). Normally, luminescence of a material is very much sensitive to the impurity concentration. However, if the impurity concentrations are too high they may act as self-quenchers (Yang et al., 1993). The IL patterns of kyanite depend on various factors such as $\mathrm{Fe}^{3+}$ ions, crystal defects, ion implantation, luminescence activators and other quenchers. The variation of IL yield in pelletized and crystalline sample is attributed to the surface roughness of the sample (Kalantaryan et al., 1997).

Photoluminescence spectra of crystalline and pelletized kyanites irradiated with $100 \mathrm{MeV}$ swift $\mathrm{Ni}^{+8}$ ions for fluences in the range $1 \times 10^{11}-1 \times 10^{14}$ ions $/ \mathrm{cm}^{2}$ followed by $442 \mathrm{~nm}$ laser beam excitation are recorded and the results obtained are presented in Figs. 3 and 4, respectively. These figures indicate a pair of sharp and intense emission with peaks at 688.7 and $705.9 \mathrm{~nm}$ along with a broad PL emission in the region $700-900 \mathrm{~nm}$. The PL intensity in all the samples studied in the present work is found to be decreased with increase of ion fluence.

The infrared absorption of as it is and ion-irradiated kyanite samples have been studied. The results revealed the presence of silicates and water even before irradiation of these samples. The characteristic absorption bands of Al-O and $\mathrm{Si}-\mathrm{O}$ are found to reduce with increase of ion fluence. The reduction in IL intensity near 775 and $875 \mathrm{~nm}$ and PL intensities in the range $700-900 \mathrm{~nm}$ in the present studies might be attributed to $\mathrm{Al}-\mathrm{O}$ and $\mathrm{Si}-\mathrm{O}$ bonds. Also, the

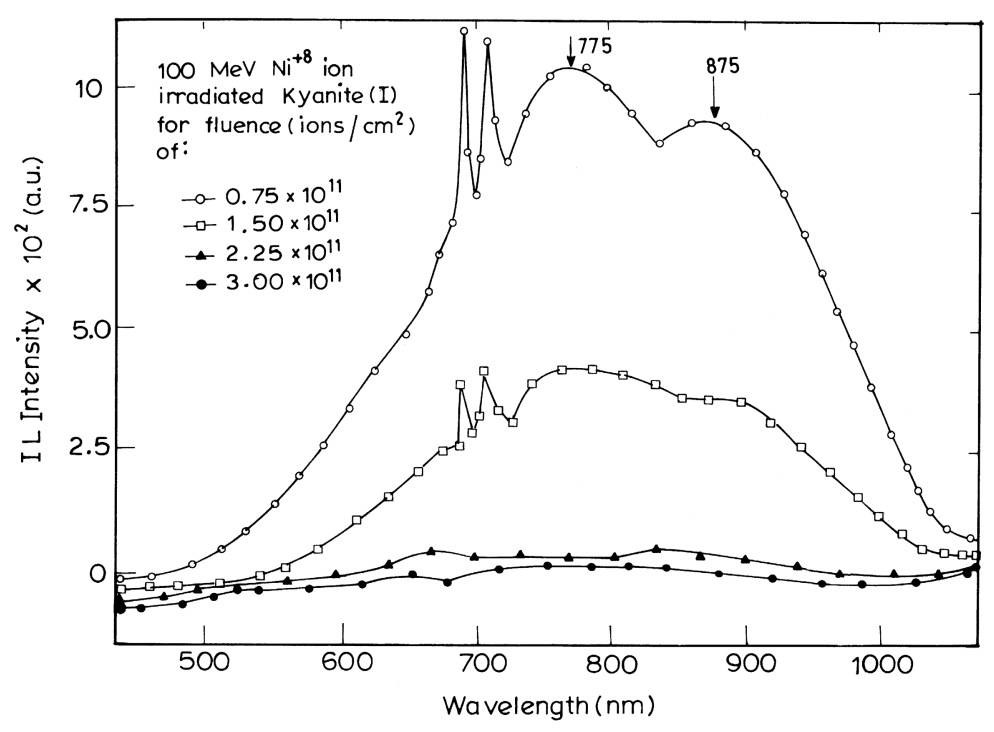

Fig. 1. Ionoluminescence spectra of Kyanite (I) irradiated with $100 \mathrm{MeV} \mathrm{Ni}{ }^{+8}$ ions. 


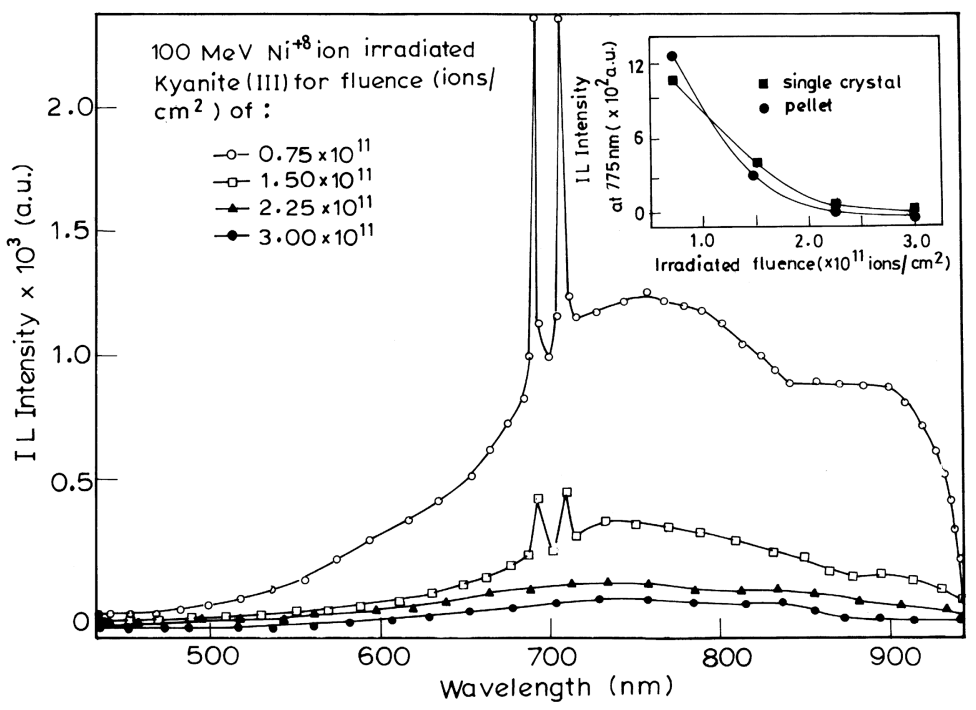

Fig. 2. Ionoluminescence spectra of Kyanite (III) irradiated with $100 \mathrm{MeV} \mathrm{Ni}{ }^{+8}$ ions.

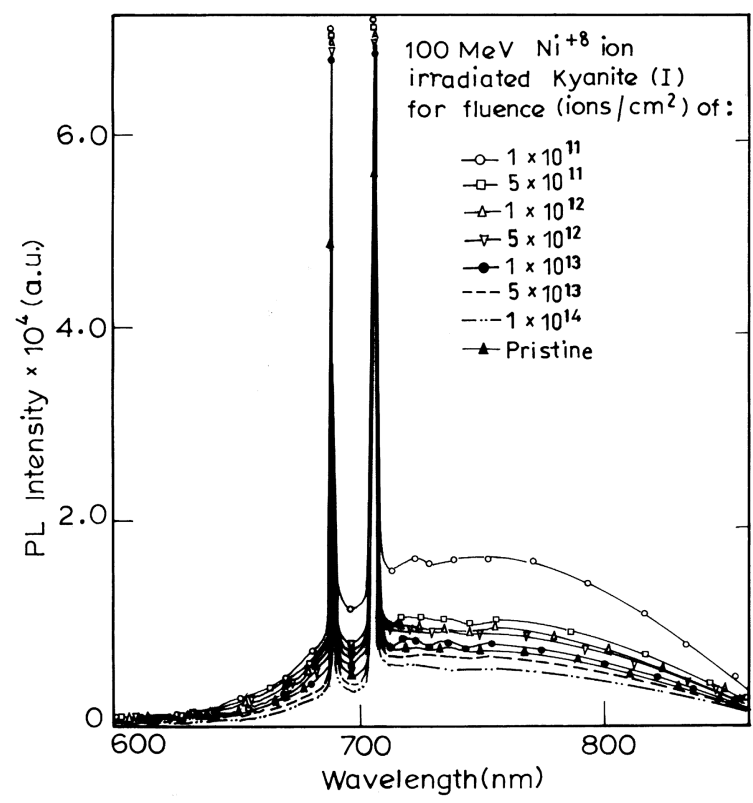

Fig. 3. Photoluminescence spectra ( $\left.\lambda_{\text {excitation }} 442 \mathrm{~nm}\right)$ of $100 \mathrm{MeV}$ $\mathrm{Ni}^{+8}$ ion-irradiated Kyanite (I) at RT.

variation in PL and IL intensity in crystalline and pelletized form of kyanite are attributed to physical nature of the sample. The sharp PL emission peaks present in both forms of kyanite samples (I and III) are attributed to $\mathrm{Fe}^{3+}$ as the same is detected from energy dispersion spectrum (EDS) and electron spin resonance (ESR) (Nagabhushana and Lakshminarasappa, 2002). The irradiation effects may lead to the restructuring of the surface chemical species because of the energy deposited through electronic loss during the

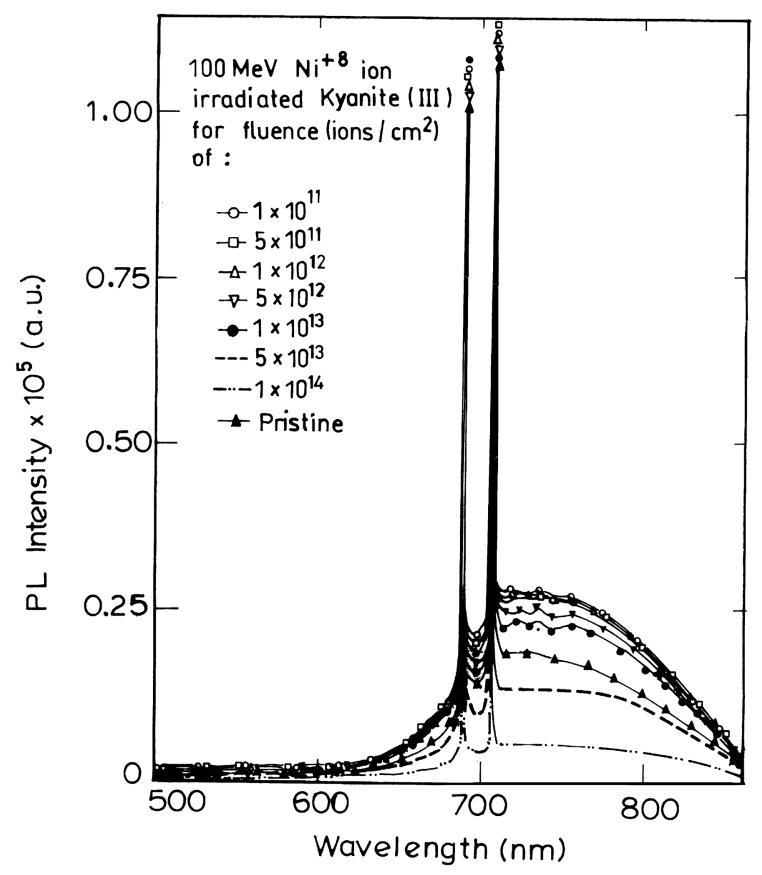

Fig. 4. Photoluminescence spectra ( $\lambda_{\text {excitation }} 442 \mathrm{~nm}$ ) of $100 \mathrm{MeV}$ $\mathrm{Ni}^{+8}$ ion-irradiated Kyanite (III) at RT.

process of swift heavy ion irradiation and, formation of ion-induced defects leading to nonradiative recombination centers. These two processes are simultaneous consequences of irradiation and they compete with each other. Enhancement or degradation in the PL and IL in the present studies might be due to the balance between these two effects (Bhave et al., 1999). 


\section{Conclusions}

The sharp IL and PL peaks observed in the range $680-720 \mathrm{~nm}$ in kyanite samples are attributed to luminescence centers activated by $\mathrm{Fe}^{3+}$ centers and, the decrease in intensity with increase of $\mathrm{Ni}^{+8}$ ion fluence is attributed to $\mathrm{Fe}^{3+}$ quenchers. The reduction of both $\mathrm{IL}$ and PL intensities in the longer wavelength region (with peaks at 775 and $875 \mathrm{~nm}$ ) with increase of $\mathrm{Ni}^{+}$ion fluence might be attributed to the destruction of $\mathrm{Si}-\mathrm{O}$ and $\mathrm{Al}-\mathrm{O}$ bonds.

\section{References}

Bhave, T.M., Hullavarad, S.S., Bhoraskar, S.V., Hegde, S.G., Kanjilal, D., 1999. FTIR studies of swift silicon and oxygen ion irradiated porus silicon. Nucl. Instrum. Methods B 156, 121-124.

Dana, E.S., Ford, E.M., 1962. Minerology Asia Publishing House, Bombay, p. 617
Homman, N.P.O., Yang, C., Malmqvist, K.G., 1994. A highly method for rare-earth element analysis using ionoluminescence combined with PIXE. Nucl. Instrum. Methods A 353, 610-614.

Kalantaryan, O.V., Kolesnik, V.T., Kononenko, S.I., Muratov, V.I., Storizhko, V.E., 1997. Silicon luminescence induced by irradiation with fast hydrogen ions. Nucl. Instrum. Methods B 129, 77-79.

Malmqvist, K.G., Elfman, M., Remond, G., Yang, C., 1996. PIXE and Ionoluminescence-A synergetic Analytical Combination. Nucl. Instrum. Methods B 109/110, 227-233.

Nagabhushana, H., Lakshminarasappa, B.N., 2002. Thermostimulated luminescence of aluminum silicate polymorphs. Proceedings of National Seminar on Luminescence and its Applications, Jabalpur, India, pp. 81-85.

Singh, F., Avasthi, D.K., Ravikumar, Kanjilal, D., 2001. In-situ photoluminescence and on-line ionoluminescence set up and preliminary studies at NSC. Proceedings of National Seminar on Luminescence and its Applications, Hyderabad, India, pp. 96-105.

Yang, C., Larsson, N.P.O., Swietlick, E., Malmqvist, K.G., 1993. Imaging with Ionoluminescence (IL) in a Nuclear Microprobe. Nucl. Instrum. Methods B 77, 188-194. 